\title{
Whole spine MRI is not required in investigating uncomplicated paediatric lumbosacral lipoma. A retrospective single-institution review
}

\author{
Hugo Layard Horsfall ${ }^{1,2} \cdot$ Aswin Chari $^{1,3}$ (D) Terhi Huttunen $^{1,4} \cdot$ Clare Simcock $^{5} \cdot$ Felice D'Arco $^{5} \cdot$ Dominic Thompson $^{1}$
}

Received: 10 July 2019 / Accepted: 4 September 2019 / Published online: 9 September 2019

(C) The Author(s) 2019

\begin{abstract}
Purpose Lumbosacral lipoma (LSL) is a severe occult spinal dysraphism, frequently associated with neurological, urological and orthopaedic complications. Whole spine imaging is typically performed to identify concomitant, but spatially separate, congenital anomalies. Our hypothesis: the incidence of additional, clinically significant abnormalities of the neuraxis is low; thus, imaging should be optimised at the lumbosacral region. We aim to assess the prevalence and relevance of LSL-associated lesions. Method A single-centre, retrospective, radiological review using a prospectively maintained operative database. Inclusion criteria: children ( $<16$ years) with confirmed diagnosis of LSL and received whole spine MRI. Fatty filum, syndromic cases and cutaneous stigmata above lesion level were excluded. Data was extracted from radiological imaging, reports and clinical correspondence.

Results One hundred twelve patients (40:72, M:F) aged 0.5 years (0.2-2.7) (median \pm IQR) with LSL had whole spine MRI between 2001 and 2017. Classification of LSL: transitional 48 (43\%); dorsal 30 (27\%); caudal 28 (25\%) and chaotic 6 (5\%). Additional anomalies included syringohydromyelia 44 (39\%), subcutaneous tract 19 (17\%), abnormal vertebral segmentation 18 (16\%), dermoid cyst 1 and 1 Chiari I deformity. There were no Chiari II malformations. No child required surgery for an associated lesion. Binary logistic regression revealed no factors associated with predicting secondary lesions.

Conclusions In congenital LSL, additional anomalies of the neuraxis are typically loco-regional rather than pan-CNS and additional lesions are rarely clinically significant. The loco-regional distribution of anomalies suggests that only lumbosacral spinal imaging is required in the initial evaluation of LSL. Such a policy would lessen the anaesthetic/sedation time for children and reduce imaging cost per patient. MRI protocols could be refined to optimise imaging quality at the region of interest.
\end{abstract}

Keywords Lumbosacral lipoma $\cdot$ Magnetic resonance imaging $\cdot$ Spinal dysraphism $\cdot$ Paediatric neurosurgery

Aswin Chari

aswinchari@gmail.com

1 Department of Neurosurgery, Great Ormond Street Hospital for Children, London, UK

2 Division of Neurosurgery, Department of Clinical Neurosciences, Addenbrooke's Hospital, University of Cambridge, Cambridge, UK

3 Institute of Child Health, University College London, London, UK

4 Department of Neurosurgery, Kuopio University Hospital, Kuopio, Finland

5 Department of Radiology, Great Ormond Street Hospital for Children, London, UK

\section{Introduction}

Lumbosacral lipoma (LSL) is one of the more severe forms of occult spinal dysraphism and may be associated with neurological, urological and orthopaedic complications [1-4]. In contrast to the spectrum of open neural tube defects (including myelomeningocoele), the abnormality is typically locoregional rather than pan central nervous system (CNS) [5]. Hydrocephalus and the Chiari II malformation are rarely seen in association with LSL or other occult dysraphic states $[6,7]$, which is explained by the differing embryological origins of these dysraphic abnormalities [1, 4].

Magnetic resonance imaging (MRI) remains the definitive post-natal diagnostic modality $[1-3,8,9]$; however, practice varies with regard to whether whole spine (or, indeed whole neuraxial) MRI or more dedicated lumbosacral spinal imaging 
is performed [10]. The justification for whole spine MRI is the detection of clinically occult and spatially separate abnormalities in the spine, or intracranially such as the Chiari II malformation and associated hydrocephalus. Whilst the concurrence of different dysraphic anomalies in the same patient is recognised, such cases are rare, and it is debatable whether the detection of asymptomatic, subclinical anomalies will alter surgical management. Whole spine MRI therefore has been traditionally used as a CNS 'screening tool'. There are logistic and practical implications for whole spine MRI imaging in infancy and childhood including prolonged sedation/ anaesthetic and MR scanning time [8,9]. Furthermore, whole spine imaging often reduces the quality and number of sequences dedicated to lumbosacral region which is important for surgical planning.

To our knowledge, there is no published data from larger cohorts to justify the utility of whole spine MRI over dedicated lumbosacral MRI in the evaluation of LSL. The aims of this study were twofold; firstly, we aimed to perform a singleinstitution retrospective review of whole spine imaging in cases of LSL to ascertain the incidence of concurrent spinal developmental anomalies; secondly, we assessed the anaesthetic and cost implications of whole spine versus locoregional imaging in this condition.

\section{Methods}

\section{Study design}

Retrospective chart and imaging review of patients identified from a prospectively maintained operative database.

\section{Patients}

The prospectively maintained electronic neurosurgical operative database of Great Ormond Street Hospital, London (GOSH) was used to identify all patients between 2001 and 2017. Only patients with a confirmed diagnosis of LSL and who had whole spine MRI performed and available for review were included. Other dysraphic abnormalities including thickened filum, myelocystocele and split cord malformation were excluded. Further exclusion criteria included children with an underlying syndromic diagnosis, for example Currarino syndrome and VACTERL association (vertebral defects, anal atresia, cardiac defects, tracheo-esophageal fistula, renal anomalies and limb abnormalities) given their propensity for concomitant congenital anomalies. Specifically, the vertebral segmentation anomalies in VACTERL association may require longer-term follow-up to assess for the risk of kyphoscoliotic abnormalities and were therefore excluded from this cohort. Furthermore cases with additional cutaneous stigmata of spinal dysraphism above the level of the lumbosacral lipoma were also excluded as these findings would mandate whole spine imaging. All available radiological images, neuroradiological reports and clinical correspondence were reviewed. Patient demographic characteristics were recorded including age at scan and sex.

\section{MR imaging}

MR imaging was performed using the GOSH Standard Protocol [11] with a $1.5 \mathrm{~T}$ clinical MR system (Gyroscan Intera, Philips Medical System, Best, The Netherlands), and using non-contrast whole spine sagittal and axial T1- and T2weighted sequences.

\section{Lipoma classification}

Morphological type of LSL was classified according to the Pang classification [4], which is a modification of the Chapman classification based on $\mathrm{x}$-ray myelography before advent of MRI [12]. Lipomas are classified based on their location and anatomy, distal conus and/or nerve root involvement. Other classifications used include Arai [13], which is commonly used in Japan [14]. We chose to use the same classification as our previous series [15], grouping the lipomas into simple (dorsal/caudal) and complex (transitional/chaotic). Simple fatty filum is not included in the lipoma classification.

\section{Review of imaging}

Imaging, reports and clinical documentation were reviewed with a paediatric neuroradiologist (FD) to determine the presence of any other abnormalities of the spine or neuraxis on the whole spine MRI. Specifically, we noted the presence of syringohydromyelia, low position of the cerebellar tonsils, occult dysraphisms and vertebral segmentation anomalies. For the purposes of this study, the term syringohydromyelia was used to include intramedullary cavities with MR characteristics of CSF. No attempt was made to further distinguish between hydromyelia and syrngomyelia.

\section{Statistical analysis}

Statistical analysis was performed using binary logistic regression via Statistical Package for the Social Sciences (SPSS) version 25 (IBM, Armonk, New York, USA).

\section{Cost-benefit analysis}

Total cost of the MRI examinations were calculated using an internal mechanism encompassing calculation of a combination of direct and indirect costs by the GOSH Lead Radiographer (CS). These include a breakdown of pay and associated time to perform tasks associated with these scans. 
For example: radiographer, radiologist, anaesthetic and nursing costs. In addition, non-pay costs are included including costs for consumables.

\section{Results}

A total of 347 patients with LSL were identified from the prospectively maintained neurosurgical operative database, of which 129 (37\%) patients had whole spine MRI. Twelve patients $(9 \%)$ were excluded as there was a diagnosis of fatty filum terminale and $5(4 \%)$ because MRI images were no longer available in our PACS system (Fig. 1).

Altogether, 112 patients had LSL and received whole spine MRI between 2001 and 2017 (Table 1). Our cohort contained 72 females $(64 \%)$. The median age was 0.5 years (IQR $0.2-$ 2.7 ) at time of MRI scan. The most common type of LSL was transitional $48(43 \%)$, followed by dorsal $30(27 \%)$, caudal 28 $(25 \%)$ and $6(5 \%)$ chaotic lipoma.

\section{MRI findings (Table 1)}

In addition to the LSL, the most common additional neuroradiological finding was syringohydromyelia $(n=44,39 \%)$ (Table 1 ). The majority of the syringohydromyelia were located solely within the lumbar vertebral region $(n=33 / 44,75 \%)$. Eight patients had syringohydromyelia that bridged from thoracic to lumbar vertebral levels $(n=8 / 44,18 \%)$, whilst 2 patients had the anomaly spanning from the lumbar to sacral region $(n=2 / 44,5 \%)$. One patient had two separate syringohydromyelic cavities between vertebral levels T4-8 and L3-S1.

One patient had a Chiari I deformity. Chiari II malformation and associated hydrocephalus were not seen in any of the cases.

Other anomalies commonly associated with LSL were also identified, including subcutaneous tracts, which was present in 19 patients $(n=19 / 112,17 \%)$. One case was unusual and
Table 1 Summary of lumbosacral lipoma MRI findings and patient demographics who received whole spine MRI in the initial work-up of lumbosacral lipoma

\begin{tabular}{ll}
\hline Total number of lumbosacral lipoma & 112 \\
Age (median \pm IQR) & $0.5(0.2-2.7)$ \\
Sex (M:F) & $40: 72$ \\
Lipoma type & \\
Dorsal & $30(27 \%)$ \\
Caudal & $28(25 \%)$ \\
Transitional & $48(43 \%)$ \\
Chaotic & $6(5 \%)$ \\
Any additional abnormality & $57(51 \%)$ \\
Syringohydromyelia & $44(39 \%)$ \\
Subcutaneous tract & $19(17 \%)$ \\
Vertebral segmentation anomaly & $18(16 \%)$ \\
Dermoid cyst & 1 \\
Chiari I & 1 \\
\hline
\end{tabular}

associated with a dermoid cyst ( $n=1 / 112$, Fig. 2$)$. All 19 cases of subcutaneous tracts were located within the lumbosacral region, most commonly at $\mathrm{L} 4 / 5$ or $\mathrm{L} 5 / \mathrm{S} 1$.

There were 18 instances of vertebral segmentation anomaly (16\%) (Fig. 3). The most common was segmental dysgenesis of the vertebral body $(n=13 / 18,72 \%)$. There was also fusion or partial fusion $(n=2 / 18,11 \%)$, hemivertebrae $(n=2 / 18$, $11 \%)$ and hypoplasia $(n=1 / 18,6 \%)$. These anomalies were located in the lumbosacral region $(n=17 / 18,94 \%)$, with only one segmentation anomaly in the thoracic region $(n=1 / 18$, $6 \%$ ).

None of these abnormalities required additional surgical or medical intervention and did not change the operative approach to the LSL.

Binary logistic regression was performed to ascertain whether demographic factors (age, sex) and lipoma type could predict additional abnormalities identified on the MRI or any specific abnormalities (syringohydromyelia, other dystrophic
Fig. 1 Flowchart showing the identification of lumbosacral lipoma patients who had whole spine MRI at Great Ormond St Hospital

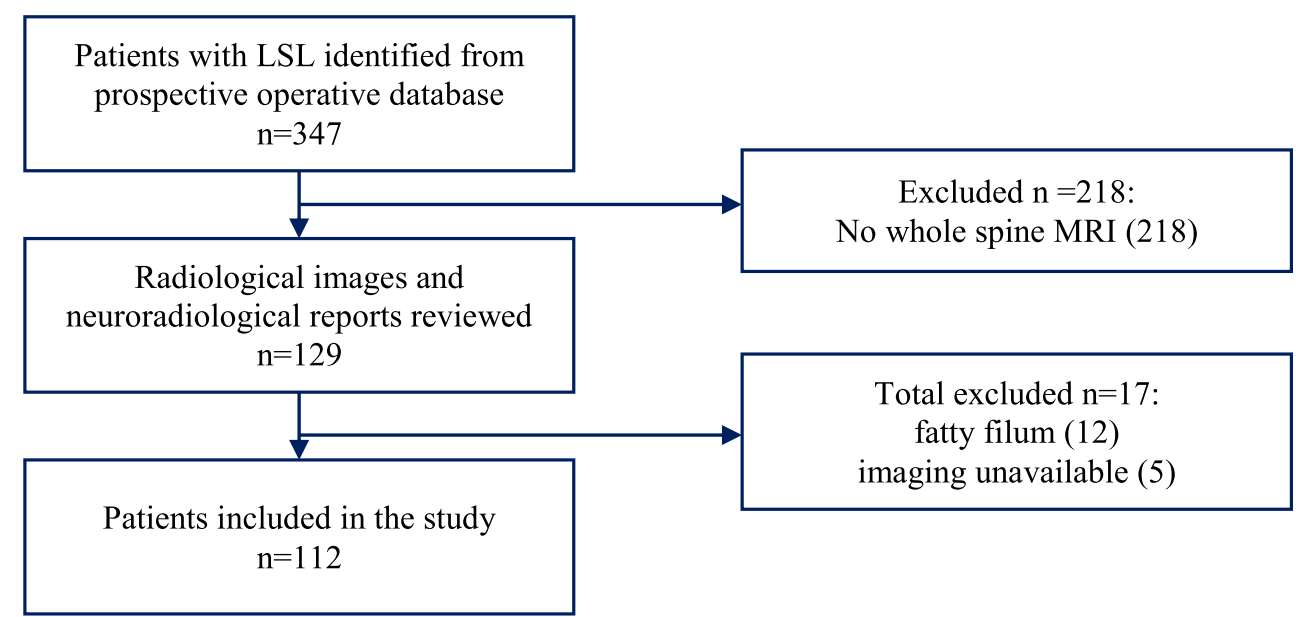


Fig. 2 Whole spine sagittal T1(a) and T2-weighted (b) MRI showing the single unusual case of a dorsal lipoma with subcutaneous tract (blue arrows) and associated dermoid cyst. Note poor spatial resolution as whole spine sequence

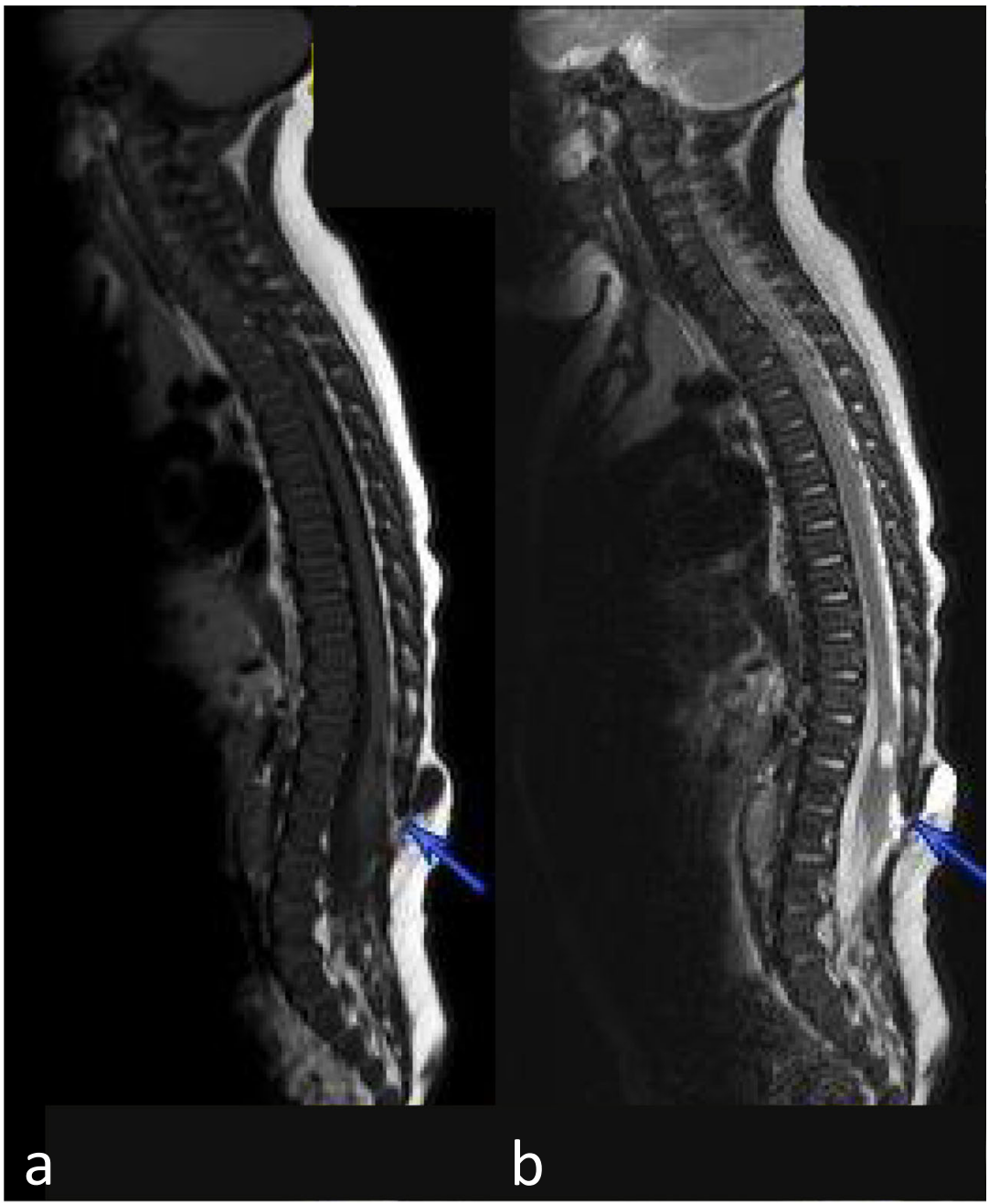

abnormalities or vertebral segmentation anomalies). All the binary logistic regression models were statistically not significant, and none of these factors were significantly predictive of general or specific additional abnormalities on the MRI whole spine.

\section{Cost benefit assessment}

Whole spine MRI required 40 min of scanning time versus $20 \mathrm{~min}$ in lumbar spine MRI (Table 2). The total cost associated with whole spine MRI and general anaesthesia is $£ 282.39$ whilst lumbar spine MRI and general anaesthesia cost $£ 197.07$. Whole spine MRI required double the amount of time and cost an additional $£ 85.32$ per scan.

\section{Discussion}

The incidence of LSL is estimated to be ranging 0.3 and 0.6 per 10,000 live births $[16,17]$. In spite of this apparent rarity, LSL is one of the more common closed dysraphic states to present to the paediatric neurosurgeon. MRI is the imaging modality of choice for LSL; however, whether it is necessary to image the entire spine (and perhaps brain) in search of associated anomalies remains unclear. This is in contrast to open dysraphic states (myelomeningocele) that are recognised to be pan-CNS disorders with a frequent concurrence of hydrocephalus and Chiari type II malformation [5].

In our cohort of 112 lumbosacral lipomas, there were no clinically significant additional abnormalities identified beyond the lumbosacral region. Syringohydromyelia was the most common associated finding, with the majority always immediately adjacent to the lumbosacral lipoma and therefore would have been detected if only dedicated lumbosacral spine imaging had been performed. This series demonstrates syringohydromyelia was present in $39 \%$ of patients. This is higher than the reported $5-25 \%$ in the literature [18-21]. It is noted that a number of syringohydromyelic cavities were either thoracolumbar (proximal extent would not be identified by the lumbosacral MRI scan) or thoracic (would not be identified at all). In our experience, these are secondary to the lipoma and often resolve following surgical resection of the 
Fig. 3 Whole spine sagittal (a) and axial (b) T2-weighted showing L3 vertebral segmentation anomaly with defective posterior elements. Note poor spatial resolution as whole spine sequence

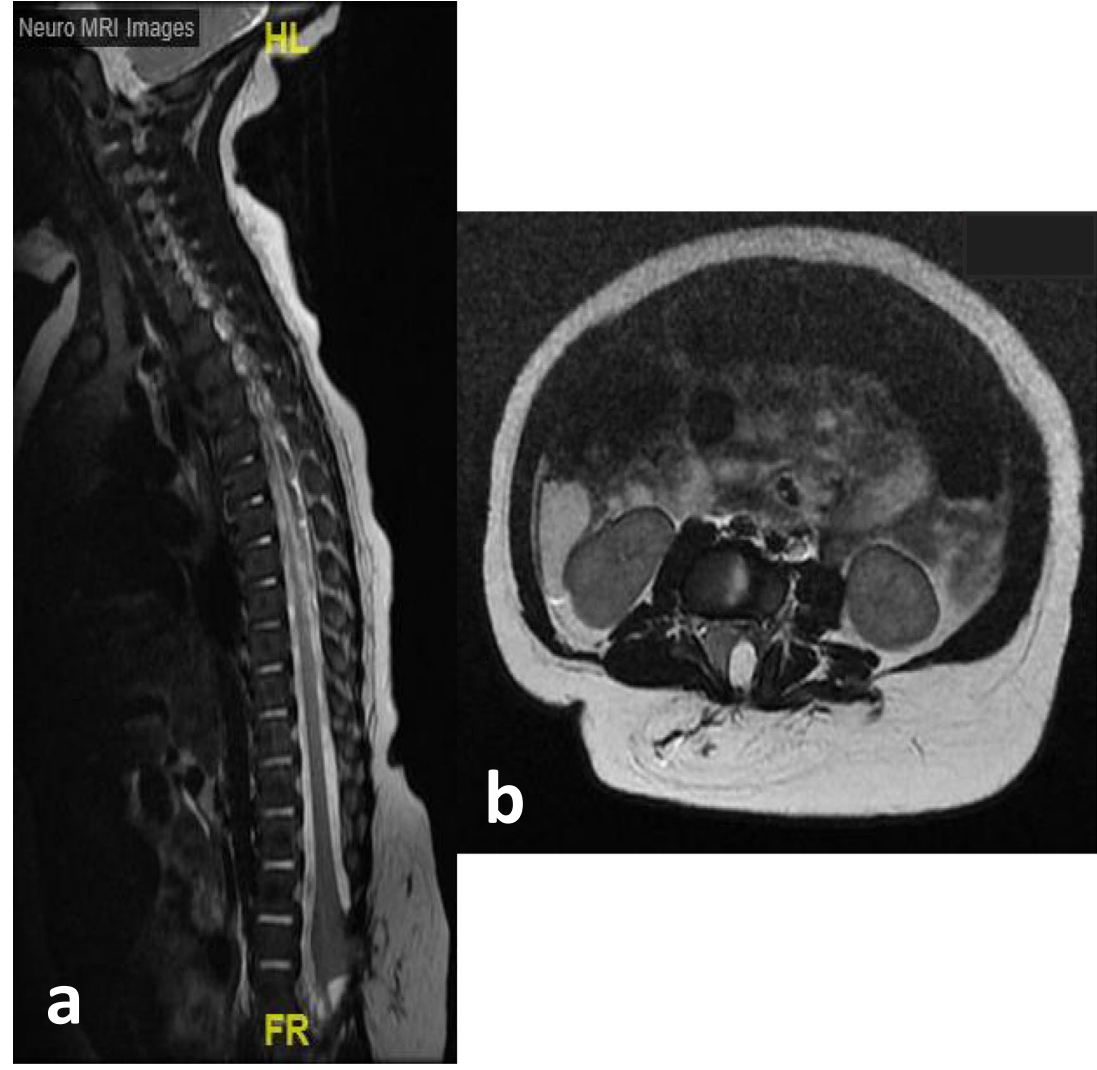

lipoma; none of those identified in this series required any specific intervention. If such a lesion is identified, we would not recommend scanning further up the spine in the immediate context, and would instead recommend scanning the rest of the spine at follow-up only in the case of persistent or worsening symptoms.

Segmentation anomalies were also present (Fig. 3). The majority of these anomalies were in the lumbosacral region and therefore would have been identified in dedicated regional imaging of the lumbosacral spine. One segmentation anomaly was outside the region of interest but in the absence of deformity would not be considered clinically relevant. In addition, thoracic segmentation anomalies are identifiable on the chest $\mathrm{x}$-ray, which is often performed prior to surgical intervention and this can be used as a screening tool if necessary. The fact that none of these segmentation anomalies altered the surgical management, however, suggests that even this x-ray may not be necessary.

Table 2 Summary of cost-benefit analysis

\begin{tabular}{llcl}
\hline MRI & Time (min) & Unit cost (£) & Unit Cost + GA (£) \\
\hline Whole spine & 40 & 228.69 & 282.39 \\
Spine lumbar & 20 & 146.16 & 197.07 \\
Benefit & 20 & 82.53 & 85.32 \\
\hline
\end{tabular}

This study in addition confirms that Chiari I and II are not a feature of this condition. There were no detected incidences of Chiari II and one incidence of Chiari I. The incidence of Chiari I has been reported as $1 \%$, which is similar to the incidence in our data set [22-24] and, importantly, this did not require intervention or change the management of the LSL.

Lastly, performing dedicated locoregional imaging reduces the MR time (20 min vs $40 \mathrm{~min}$ ) and provides a cost saving of $£ 82.53$ (Table 2). The time efficiency enables more patients to be scanned in 1 day and may allow the use of additional sequences such as three-dimensional constructive interference in steady state (CISS) that can be used for better surgical planning in complex cases.

Our data confirms the incidence of secondary lesions associated with uncomplicated LSL may be as high as $51 \%$. However, the majority of associated anomalies are anatomically closely related to the LSL and therefore, would be detected on regional imaging. In the absence of cutaneous stigmata or clinical signs suggesting concomitant dysraphic anomalies, our data suggest that truly occult second lesions are unlikely to be of clinical significance. Therefore, in the initial presentation and work-up of uncomplicated LSL, regional imaging is sufficient and appropriate. This yields the benefit of reduced scanning time and cost savings, in addition to a superior anatomical delineation in a smaller field of view that permits better surgical planning.

The limitations of the present study include its retrospective nature. At our institution, the protocol for MR scanning 
usually requires children under the age of four to be anaesthetised to reduce movement and artefact. With the median age at scan being 0.5 years, these children would have been anaesthetised and therefore it seems reasonable to ensure the entire spine is imaged for completeness. That being said, telling parents about additional, incidental and not clinically relevant adds anxiety to the clinical context, particularly when the surgical management is not altered. In addition, there are new fast sequences and methods to reduce the use of anaesthesia which will favour the use of a shorter protocol. There remain situations where whole spine MRI is still required including syndromes associated with dysraphism, such as VACTERL, where other spinal anomalies are common; presence of cutaneous stigmata over the upper spine; or neurological symptoms and signs suggestive of a higher lesion.

\section{Conclusions}

This study confirms the locoregional nature of complex lumbosacral lipoma and the low incidence of clinically relevant additional spinal lesions. We suggest that MRI dedicated to the lumbosacral spine is sufficient in the evaluation of lumbosacral lipoma. Local MRI permits optimal imaging quality at the region of interest which is essential in the evaluation of surgical anatomy, may reduce the anaesthetic/sedation time for children, the imaging cost per patient and allows addition of extra sequences in the region of interest when necessary.

Funding information Aswin Chari is supported by a Great Ormond Street Hospital (GOSH) Children's Charity Surgeon Scientist Fellowship and the National Institute for Health Research GOSH Biomedical Research Centre.

\section{Compliance with ethical standards}

Conflict of interest There is no conflict of interest to report.

Ethical approval Ethical approval was not required for this retrospective imaging-based study.

Informed consent Due to the retrospective long-term nature of this study, informed consent was not obtained.

Open Access This article is distributed under the terms of the Creative Commons Attribution 4.0 International License (http:// creativecommons.org/licenses/by/4.0/), which permits unrestricted use, distribution, and reproduction in any medium, provided you give appropriate credit to the original author(s) and the source, provide a link to the Creative Commons license, and indicate if changes were made.

\section{References}

1. Thompson D (2014) Spinal dysraphic anomalies; classification, presentation and management. Paediatr Child Health 24(10):431-438
2. Sarris C, Tomei K, Carmel P, Gandhi C (2012) Lipomyelomeningocele: pathology, treatment, and outcomes. Neurosurg Focus 33(4):E3

3. Finn M, Walker M (2007) Spinal lipomas: clinical spectrum, embryology, and treatment. Neurosurg Focus 23(2):1-12

4. Pang D (2019) Surgical management of complex spinal cord lipomas: how, why and when to operate. A review. J Neurosurg Pediatr 23:537-556

5. Geerdink N, van der Vliet T, Rotteveel JJ, Feuth T, Roeleveld N, Mullaart RA (2012) Essential features of Chiari II malformation in MR imaging: an interobserver reliability study-part 1 . Childs Nerv Syst 28(7):977-985

6. Chiari H (1891) Ueber Veränderungen des Kleinhirns infolge von Hydrocephalie des Grosshirns1. Dtsch Med Wochenschr 17(42): $1172-1175$

7. Stevenson K (2004) Chiari type II malformation: past, present, and future. Neurosurg Focus 16(2):1-7

8. Modic M, Weinstein M (1984) Nuclear magnetic resonance of the spine. Br Med Bull 40(2):183-186

9. Norman D, Mills C, Brant-Zawadzki M, Yeates A, Crooks L, Kaufman L (1983) Magnetic resonance imaging of the spinal cord and canal: potentials and limitations. AJR Am J Roentgenol 141(6): $1147-1152$

10. Patil P, Gupta A, Kothari P, Kekre G, Gupta R, Dikshit V, Mudkhedkar K, Kesan K (2016) Immediate and long-term outcome analysis of lipomeningomyelocele repair in asymptomatic infants in a tertiary care centre. J Pediatr Neurosci 11(2):99-104

11. D'Arco F, D'Amico A, Caranci F, Di Paolo N, Melis D, Brunetti A (2014) Cerebrovascular stenosis in neurofibromatosis type 1 and utility of magnetic resonance angiography: our experience and literature review. Radiol Med 119(6):415-421

12. Chapman PH (1982) Congenital intraspinal lipomas: anatomic considerations and surgical treatment. Childs Brain 9(1):3 37-3 47

13. Arai H, Sato K, Okuda O, Miyajima M, Hishii M, Nakanishi H, Ishii H (2001) Surgical experience of 120 patients with lumbosacral lipomas. Acta Neurochir 143:857-864

14. Morioka T, Murakami N, Shimogawa T, Mukae N, Hashiguchi K, Suzuki SO, Iihara K (2017) Neurosurgical management and pathology of lumbosacral lipomas with tethered cord. Neuropathology 37(5):385-392

15. Jones V, Wykes V, Cohen N, Thompson D, Jacques TS (2018) The pathology of lumbosacral lipomas: macroscopic and microscopic disparity have implications for embryogenesis and mode of clinical deterioration. Histopathology 72(7):1136-1144

16. Agopian A, Canfield M, Olney R, Lupo P, Ramadhani T, Mitchell L et al (2012) Spina bifida subtypes and sub-phenotypes by maternal race/ethnicity in the National Birth Defects Prevention Study. Am J Med Genet A 158A(1):109-115

17. De Wals P, Van Allen M, Lowry R, Evans J, Van den Hof $M$, Crowley $M$ et al (2008) Impact of folic acid food fortification on the birth prevalence of lipomyelomeningocele in Canada. Birth Defects Res A Clin Mol Teratol 82(2):106109

18. Komiyama M, Hakuba A, Inoue Y, Yasui T, Yagura H, Baba M, Nishimura S (1987) Magnetic resonance imaging: lumbosacral lipoma. Surg Neurol 28(4):259-264

19. Taviere V, Brunelle F, Baraton J, Temam M, Pierre-Kahn A, Lallemand D (1989) MRI study of lumbosacral lipoma in children. Pediatr Radiol 19(5):316-320

20. Wykes V, Desai D, Thompson DN (2012) Asymptomatic lumbosacral lipomas - a natural history study. Childs Nerv Syst 28(10): $1731-1739$

21. Xenos C, Sgouros S, Walsh R, Hockley A (2000) Spinal lipomas in children. Pediatr Neurosurg 32(6):295-307 
22. Pierre-Kahn A, Lacombe J, Pichon J, Giudicelli Y, Renier D, Sainte-Rose C, Perrigot M, Hirsch JF (1986) Intraspinal lipomas with spina bifida. Prognosis and treatment in 73 cases. J Neurosurg 65(6):756-761

23. Meadows J, Kraut M, Guarnieri M, Haroun RI, Carson BS (2000) Asymptomatic Chiari type I malformations identified on magnetic resonance imaging. J Neurosurg 92(6):920-926
24. Aitken LA, Lindan CE, Sidney S, Gupta N, Barkovich AJ, Sorel M, Wu YW (2009) Chiari type I malformation in a pediatric population. Pediatr Neurol 40(6):449-454

Publisher's note Springer Nature remains neutral with regard to jurisdictional claims in published maps and institutional affiliations. 